\title{
Design and analysis of high-speed cam mechanism using Fourier series
}

\author{
Changjiang Zhou ${ }^{1 *}$, Bo $\mathrm{Hu}^{1}$, Siyu Chen ${ }^{2}$, Lin $\mathrm{Ma}^{3}$
}

(1. State Key Laboratory of Advanced Design and Manufacturing for Vehicle Body, Hunan University, Changsha 410082, China

2. State Key Laboratory of High Performance Complex Manufacturing, Central South University, Changsha

$$
\text { 410083, China }
$$

3. Science and Engineering Faculty, Queensland University of Technology, Brisbane 2434, Australia)

\begin{abstract}
This study proposes an innovative and comprehensive method for the design and analysis of a high-speed cam mechanism. In the proposed method, the displacement function of the cam follower is described using Fourier series. The coefficients of the series are obtained by constraint equations during the cam rise period and the minimum principle of displacement error function (where, error function refers to the variance between actual displacement and ideal displacement of the cam follower during the dwell). The validity of the proposed design method is verified through a case study with focus on high-speed cam mechanism design. In the design case, kinematic characteristics analysis of this cam follower shows that the order of continuous derivative of displacement transfer function is higher, the characteristic value of its dynamic torque is smaller, and its jerk at the impact point is smaller than some common disc cam mechanisms. The results indicate that the proposed method can reduce the vibration of the cam mechanisms and its impact velocity is smaller than the existing design methods.
\end{abstract}

Keywords: High-speed mechanism; Fourier series; Kinematic characteristics; Jerk; Impact velocity

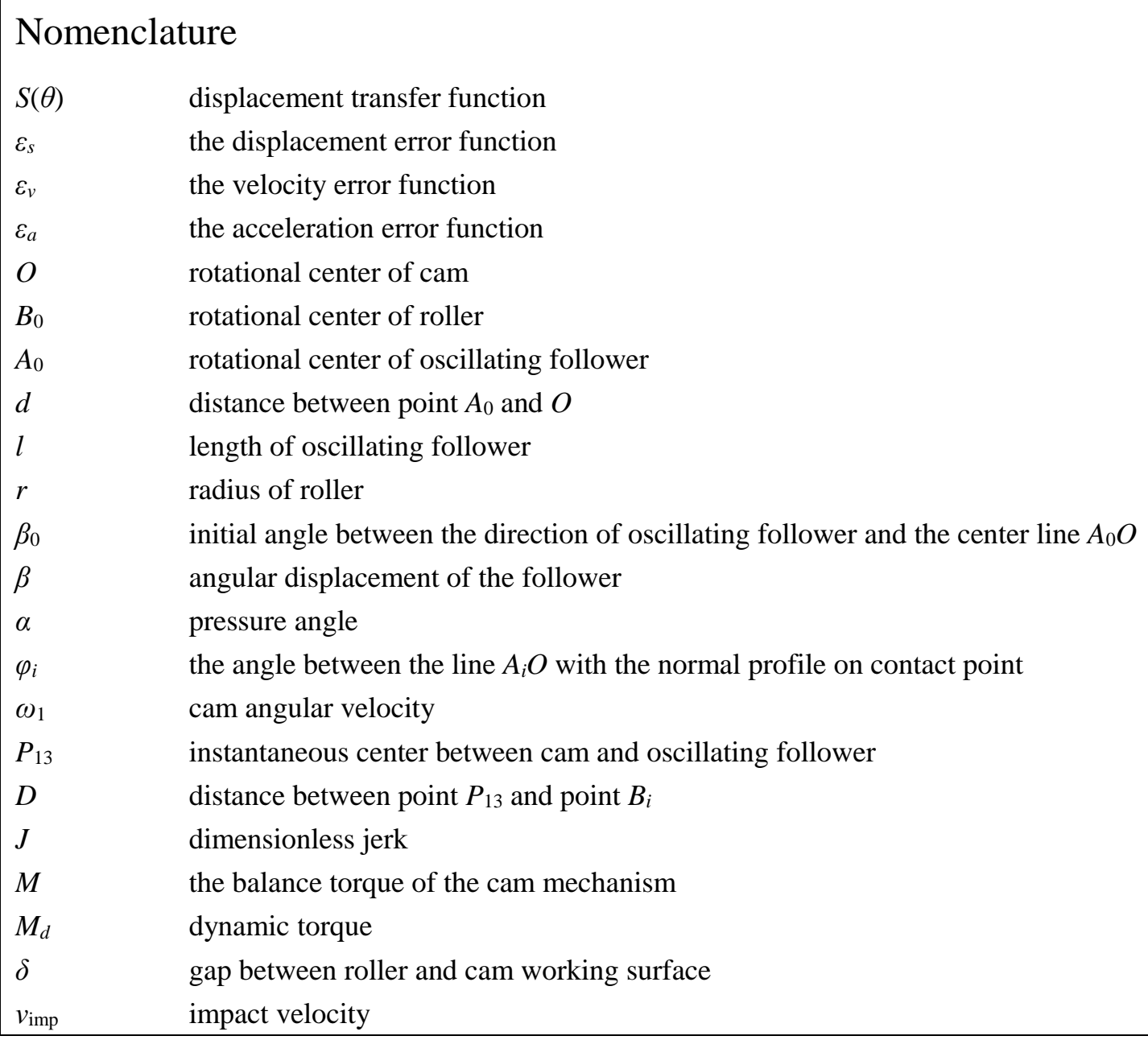




\begin{tabular}{|ll|}
\hline$J_{\text {imp }}$ & dimensionless impact jerk on crossing point \\
$j_{2}$ & rotational inertia of the follower \\
$m_{23}$ & the mass of the roller (2) and the oscillating follower (3) \\
$r_{c}$ & the distance between the center of mass and rotation center of the follower \\
$F$ & the external load of the follower \\
\hline
\end{tabular}

\section{Introduction}

In the development of high-precision and high-speed cam mechanism, its dynamic characteristics and rubbing have become a major concern of the researchers [1-4]. Hrones et al.[5] demonstrated that dynamic characteristics of the cam follower with constant acceleration were worst at high speed in terms of theoretical analysis, which was proved by Mitchell[6] with experiments. To improve the dynamic characteristics, Hiroshi[7] described the displacement function of the cam follower using the combined curve of correction sine, trapezoidal and constant velocity curve, and on this basis Peng et al.[8] optimized its kinematic characteristics. Therefore, this design method has been applied widely in mid-speed cam mechanism. Sun et al.[9] proposed an approach to designing the cam profile combining typical curves in Pro/e. Different types of spline curves were applied in the design of the cam mechanisms, and these design methodologies have improved its kinematic capability [10-13].

The dynamic characteristics of the high-speed cam mechanism were studied by many researchers, and different dynamic parameters were minimized for optimizing the cam profile. Gao et al. [14] optimized the cam profile of the zoom system based on the least square method. Cam with three circular-arc profiles was designed by Hsieh [15], and its kinematic characteristics are then investigated numerically using an equivalent slider-crank representation. Hsieh [16] obtained an analytical description of the driving cam profile using the coordinate transformation and the conjugate surface theory. Moreover, Qiu [17] and Fabien [18] improved the dynamic characteristics of the cam mechanism by optimizing the residual vibration of the cam follower. Wiederrich [19] and Gutman [20] studied dynamic and frequency of cams using finite trigonometric series, respectively. However, the detailed design method was not elaborated and the errors during dwell can not control in their papers. In addition, it is not mentioned to calculate the number of terms of the trigonometric series. Cam profile synthesis method using spline curves was well used in the design of cam profiles because of its superior controllability [21-23]. However, when the higher order of derivative for displacement curve is required continuous, the order and terms number in the spline curves increase. And more coefficients of the terms have to be determined, which causes the amount of calculation much larger.

The existing literature lacks a systematic method for the design and synthesis of high-speed cams using a Fourier series. Therefore, this research presents an innovative design approach to high-speed cam mechanism using a Fourier series and a synthetic analysis method for its dynamic characteristics. The proposed method comprises four major steps: (1) theoretical analysis of the design of a Fourier series cam mechanism; (2) Development of the displacement transfer function for the cam profile; (3) verification of the effectiveness of this approach for designing an Fourier series cam; and (4) Comparative study on the dynamic characteristics of the Fourier series cam concerning other common disc cams.

\section{Design method for a Fourier series cam}

When the cam mechanisms perform a periodic motion, the displacement function of the cam follower (hereinafter referred to displacement transfer function) can be described by one periodic function $S(\theta)$ (where $\theta$ is the rotational angle of the cam). According to the sufficient condition of the Dirichlet theory, $S(\theta)$ can be expanded as a convergent Fourier series. Namely, the function $S(\theta)$ can be expressed as 


$$
S(\theta)=\frac{a_{0}}{2}+\sum_{k=1}^{\infty}\left(a_{k} \cos (k \theta)+b_{k} \sin (k \theta)\right)
$$

where

$$
\begin{gathered}
a_{0}=\frac{1}{\pi} \int_{0}^{2 \pi} S(\theta) \mathrm{d} \theta \\
a_{k}=\frac{1}{\pi} \int_{0}^{2 \pi} S(\theta) \cos (k \theta) \mathrm{d} \theta \\
b_{k}=\frac{1}{\pi} \int_{0}^{2 \pi} S(\theta) \sin (k \theta) \mathrm{d} \theta
\end{gathered}
$$

Eq.(1) includes infinite terms, that is to say, it is composed of infinite harmonic. When $k \omega$ (the excitation frequency) is closed to $\omega_{n}$ (the natural frequency of the cam mechanism), the follower will cause resonance and the stability of the cam mechanism will be the worse. The displacement transfer function approximated by limited terms of a Fourier series is able to meet the motion accuracy requirement of the cam mechanism and avoid high order harmonic which results in the resonance. Namely, when $n \omega<\omega_{n}$ and $(n+1) \omega \geq \omega_{n} \quad($ where $\omega=\mathrm{d} \theta / \mathrm{d} t$ is the angular velocity of the cam mechanism, $\omega_{n}$ is the first order natural frequency), the number of the terms was set as $n$, which can enable the cam mechanism to avoid resonance. So the displacement transfer function of the cam designed by a Fourier series can be expressed as:

$$
S(\theta)=a_{0}+\sum_{k=1}^{n}\left(a_{k} \cos (k \theta)+b_{k} \sin (k \theta)\right)
$$

\section{Development of a displacement transfer function}

The displacement transfer function can be constructed as a Fourier series with finite terms to avoid resonance. Therefore, the actual motion and the ideal motion of the cam follower can't be completely consistent. Its error can be controlled by the variance between the actual displacement and the ideal displacement of the cam follower during the dwell period. In orde to make the cam mechanism keep sufficient precision, the error of displacement must be minimal.

In engineering design, there are strict motion accuracy requirements in some sections of the displacement curve. We assume the displacement curve is composed of $m$-sections curves with some strict motion accuracy requirements. The actual and the ideal displacement transfer function of section $i$ (where, $i=1,2,3, \cdots, m$ ) are represented by $S_{i}(\theta)$ and $\bar{S}_{i}(\theta)$, respectively. The start angular displacement and the end angular displacement of section $i$ are given by $\theta_{a_{i}}$ and $\theta_{e_{i}}$, respectively. The displacement error function, $\varepsilon_{s}$, between $S_{i}(\theta)$ and $\bar{S}_{i}(\theta)$ is calculated as follows:

$$
\varepsilon_{s}=\sum_{i=1}^{m}\left\{\int_{\theta_{a_{i}}}^{\theta_{e_{i}}} \rho_{i}\left[S_{i}(\theta)-\overline{S_{i}}(\theta)\right]^{2} \mathrm{~d} \theta\right\}
$$

where $\rho_{i}$ is the weighted factor. The accuracy of the displacement transfer function will be high during the dwell period, so $\rho_{i}$ should be large. The displacement transfer function will contain some constraint conditions in the rise and the return period. There are $p$ constraint conditions, hypothetically, $p$ coefficients of $S_{i}(\theta),\left(a_{0}, a_{1}, \cdots, a_{p-1}\right)$, will be obtained as follows:

$$
\left\{\begin{array}{c}
a_{0}=h_{0}\left(a_{p}, a_{p+1}, \cdots, a_{n-1}, a_{n}, b_{1}, b_{2}, \cdots, b_{n}\right) \\
a_{1}=h_{1}\left(a_{p}, a_{p+1}, \cdots, a_{n-1}, a_{n}, b_{1}, b_{2}, \cdots, b_{n}\right) \\
\cdots \\
a_{p-2}=h_{p-2}\left(a_{p}, a_{p+1}, \cdots, a_{n-1}, a_{n}, b_{1}, b_{2}, \cdots, b_{n}\right) \\
a_{p-1}=h_{p-1}\left(a_{p}, a_{p+1}, \cdots, a_{n-1}, a_{n}, b_{1}, b_{2}, \cdots, b_{n}\right)
\end{array}\right.
$$

Substituting Eq.(4) into Eq.(3), the displacement error function can be represented as follows:

$$
\varepsilon_{s}=\varepsilon\left(a_{p}, a_{p+1}, \cdots, a_{n-1}, a_{n}, b_{1}, b_{2}, \cdots, b_{n}\right)
$$


In order to minimize the displacement error, partial derivatives of the displacement error function for each variable must be equal to zero. Therefore, $a_{j}$ and $b_{k}$ (where, $j=p, p+1, \cdots, n ; k=1,2, \cdots, n$ ) in Eq.(5) can be calculated as follows:

$$
\left\{\begin{array}{l}
\frac{\partial \varepsilon_{s}}{\partial a_{j}}=0 \\
\frac{\partial \varepsilon_{s}}{\partial b_{k}}=0
\end{array}\right.
$$

The displacement transfer function and the displacement error function, $S(\theta)$ and $\varepsilon_{s}$, can be obtained by substituting $a_{0}, a_{k}$ and $b_{k}$ (where, $k=1,2, \cdots, n$ ) into Eq.(2) and Eq.(5), respectively.

When the cam is designed by Fourier series, there are some tiny waves during the dwell period. The larger $n$ is, the more the number of tiny waves are. However, $\varepsilon_{s}$ is related to $n$ ( $n$ refers to the items of $S(\theta)$ ). When value of $n$ is increased, the displacement error will be decreased. Therefore, $n$ should be minimum integer while the error of displacement, velocity and acceleration meeting the following inequation:

$$
\left\{\begin{array}{l}
\varepsilon_{s} \leq 10^{-4} \\
\varepsilon_{v} \leq 10^{-4} \\
\varepsilon_{a} \leq 10^{-4}
\end{array}\right.
$$

where, according to the definition of $\varepsilon_{s}$ in Eq.(3), $\varepsilon_{v}$ and $\varepsilon_{a}$ can be defined as:

$$
\left\{\begin{array}{l}
\varepsilon_{v}=\sum_{i=1}^{m}\left\{\int_{\theta_{a_{i}}}^{\theta_{c_{i}}} \rho_{i}\left[V_{i}(\theta)-\overline{V_{i}}(\theta)\right]^{2} \mathrm{~d} \theta\right\} \\
\varepsilon_{a}=\sum_{i=1}^{m}\left\{\int_{\theta_{a_{i}}}^{\theta_{c_{i}}} \rho_{i}\left[A_{i}(\theta)-\overline{A_{i}}(\theta)\right]^{2} \mathrm{~d} \theta\right\}
\end{array}\right.
$$

\section{Cam profile and pressure angle}

For manufacturing the Fourier series cam, it is necessary to calculate the cam profile according to $S(\theta)$ and structural parameters of the cam mechanism. Figure 1 shows the schematic diagram of the cam mechanism with an oscillating roller follower. This mechanism consists of a cam (1) and an oscillating follower (3) with roller (2).

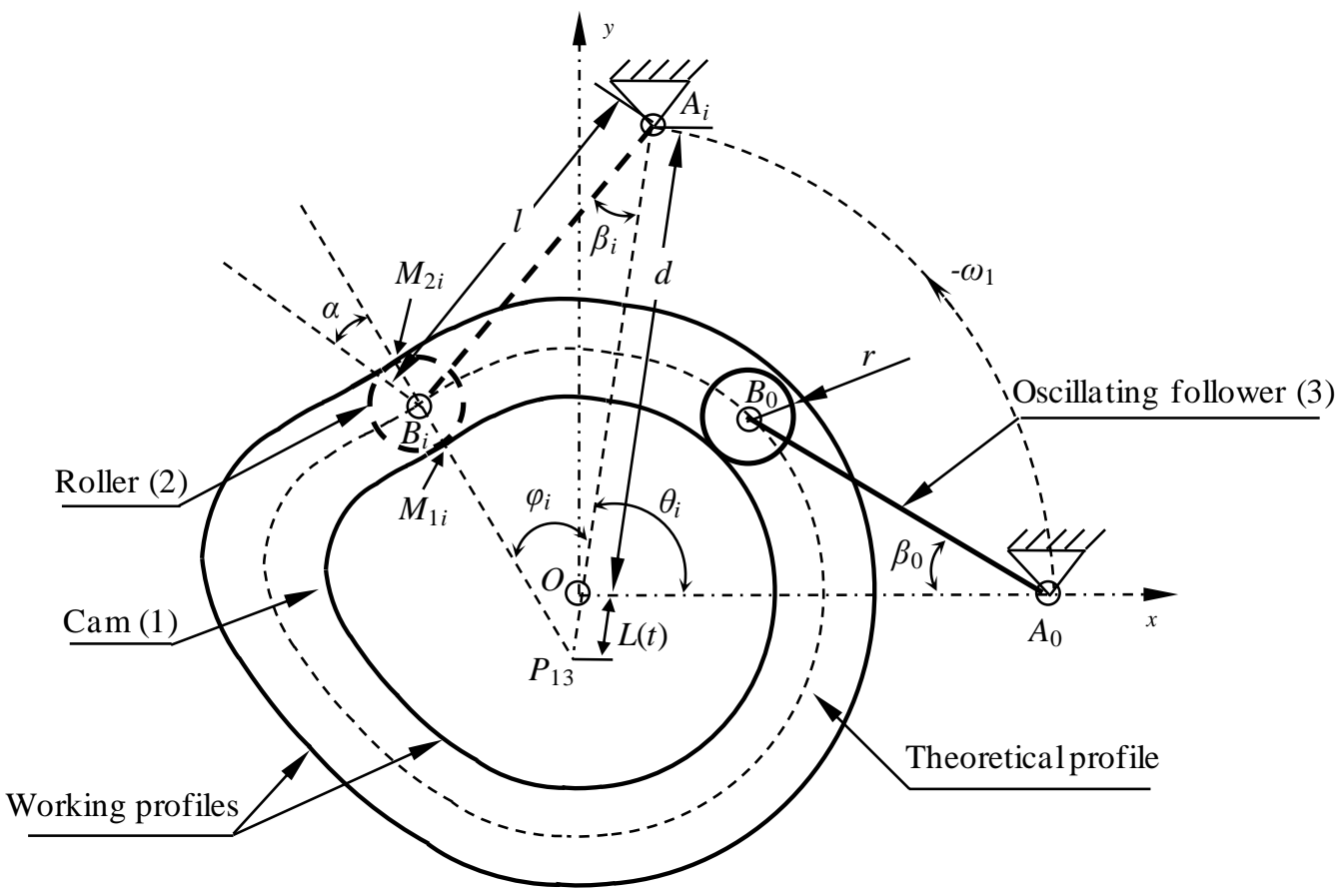

Fig.1. Schematic diagram of cam mechanism based on kinematic inversion 
$O, B_{0}$ and $A_{0}$ are the rotational center of cam (1), the rotational center of roller (2) and the rotational center of the oscillating follower (3), respectively. $\beta_{0}$ is the initial angle between the direction of the oscillating follower (3) and the center line $A_{0} O$. When the angular displacement of cam (1) is $\theta_{i}$, the state of the system is denoted as the $i$-state which is used to define the geometry of the cam-follower system. According to kinematic inversion, this $i$-state can be considered as $A_{0}$ rotation of $-\theta_{i}$ about pivot $O$. The trajectory of $B_{i}$ is the theoretical profile of the cam. It can be written as:

$$
\begin{gathered}
x_{i}=d \cos \theta_{i}-l \cos \left(\theta_{i}-\beta_{i}\right) \\
y_{i}=d \sin \theta_{i}-l \sin \left(\theta_{i}-\beta_{i}\right)
\end{gathered}
$$

Here, $\beta_{i}=\beta_{0}+S\left(\theta_{i}\right)$, where $d$ is the distance between point $A_{0}$ and $O ; l$ and $r$ are the length of oscillating follower and the radius of roller, respectively. The working profiles of the cam (the trajectory of contact point $M_{1 i}$ and $M_{2 i}$ ) can be obtained by the following equations.

$$
\begin{aligned}
& x_{1,2}=x_{i} \pm r \cos \left(\varphi_{i}+\theta_{i}\right) \\
& y_{1,2}=y_{i} \pm r \sin \left(\varphi_{i}+\theta_{i}\right)
\end{aligned}
$$

where $\varphi_{i}$ is the angle between the line $A_{i} O$ with the normal profile of the cam on contact point. It is defined as follows:

$$
\varphi_{i}=\arcsin \frac{l \sin \beta_{i}}{D}
$$

where $D$ is the distance between the instantaneous center of rotation $P_{13}$ and point $B_{i}$. It is defined by the following equation:

$$
D=\sqrt{l^{2}+(d+L(t))^{2}-2 l(d+L(t)) \cos \beta_{i}}
$$

with

$$
L(t)=\frac{d \cdot \dot{\beta}_{i}}{\omega_{1}-\dot{\beta}_{i}}
$$

where $\dot{\beta}_{i}$ is the angular velocity of the oscillating follower (3) in $i$-state. According to data of the working profiles, the Fourier series cam can be manufactured.

The distribution of forces allows a much larger limiting pressure angle, because the cam mechanism is virtually impossible to jam the oscillating roller follower. Thus, with an oscillating follower, a smaller cam may be used. However, the pressure angle should be controlled for best performance. In $i$-state, it is defined by:

$$
\alpha_{i}=\frac{\pi}{2}-\beta_{i}-\phi_{i}
$$

\begin{tabular}{|c|c|c|c|c|c|c|}
\hline$d(\mathrm{~mm})$ & $l(\mathrm{~mm})$ & & $\beta_{0}(\mathrm{rad})$ & & \multicolumn{2}{|c|}{$r(\mathrm{~mm})$} \\
\hline 50 & 40 & & $\pi / 4$ & & \multicolumn{2}{|c|}{5.5} \\
\hline Cam a & & $0 \sim 0.5 \pi$ & $0.5 \pi \sim \pi$ & $\pi$ & $\pi \sim 1.5 \pi$ & $1.5 \pi \sim 2 \pi$ \\
\hline Follower & & 0 & $0 \sim \pi / 8$ & $\pi / 8$ & $\pi / 8 \sim 0$ & 0 \\
\hline
\end{tabular}

\section{Case study and discussion}

To verify the validity of the innovative design method proposed in this study, a Fourier series cam mechanism with an oscillating roller follower was designed. The rotational speed of the cam is $3500 \mathrm{rmin}^{-1}$. The structural parameters of this cam mechanism are showed in Table 1, and the motion law of the follower is showed in Table 2.

Table 1 Structural parameters of cam mechanism 


\subsection{Design case}

The follower characteristics should be normalized (dimensionless) for comparative analysis of the performance of cam with different cam curves. During the dwell period $(0 \leq \theta \leq 0.5 \pi \cup 1.5 \pi \leq \theta \leq 2 \pi), \overline{S_{i}}(\theta)=0$ (where, $\overline{S_{i}}(\theta)$ is the ideal displacement transfer function). The equality constraints in this design case can be expressed as:

$$
\left\{\begin{array}{c}
S(0.5 \pi)=S(1.5 \pi)=0 \\
S(\pi)=1 \\
\dot{S}(\pi)=0
\end{array}\right.
$$

By substituting $\overline{S_{i}}(\theta)=0(0 \leq \theta \leq 0.5 \pi \cup 1.5 \pi \leq \theta \leq 2 \pi)$ into Eq.(3), the displacement error function of this design case can be written as:

$$
\varepsilon_{s}=\int_{0}^{0.5 \pi} S(\theta)^{2} \mathrm{~d} \theta+\int_{1.5 \pi}^{2 \pi} S(\theta)^{2} \mathrm{~d} \theta
$$

where, $\rho_{i}=1$. Combining Eqs.(7), (8) with Eq.(18), inequality constraints can be expressed as:

$$
\left\{\begin{array}{c}
\varepsilon_{s}=\int_{0}^{0.5 \pi} S(\theta)^{2} \mathrm{~d} \theta+\int_{1.5 \pi}^{2 \pi} S(\theta)^{2} \mathrm{~d} \theta \leq 10^{-4} \\
\varepsilon_{v}=\int_{0}^{0.5 \pi}\left(\frac{\mathrm{d} S(\theta)}{\mathrm{d} t}\right)^{2} \mathrm{~d} \theta+\int_{1.5 \pi}^{2 \pi}\left(\frac{\mathrm{d} S(\theta)}{\mathrm{d} t}\right)^{2} \mathrm{~d} \theta \leq 10^{-4} \\
\varepsilon_{a}=\int_{0}^{0.5 \pi}\left(\frac{\mathrm{d}^{2} S(\theta)}{\mathrm{d} t^{2}}\right)^{2} \mathrm{~d} \theta+\int_{1.5 \pi}^{2 \pi}\left(\frac{\mathrm{d}^{2} S(\theta)}{\mathrm{d} t^{2}}\right)^{2} \mathrm{~d} \theta \leq 10^{-4}
\end{array}\right.
$$

By substituting Eq.(2) and (17) into Eq.(18), the displacement error function can be written as $\varepsilon_{s}=\varepsilon\left(a_{k}, b_{k}\right)(k=2,3, \cdots, n) . a_{k}$ and $b_{k}$ can be obtained as follows:

$$
\frac{\partial \varepsilon_{s}}{\partial a_{k}}=\frac{\partial \varepsilon_{s}}{\partial b_{k}}=0(k=2,3, \cdots, n)
$$

Taking into equality constrains (Eq.(17)) and inequality constrains (Eq.(19)) account, the minimum integer $n$ is equal to 7 calculated by Genetic Algorithm. By substituting coefficient $a_{0}, a_{k}$ and $b_{k}(\mathrm{n}=1, \cdots, 7)$ calculated by Eqs.(16) and (18) into Eq.(15), the displacement transfer function, $S(\theta)$, is expressed as:

$$
S(\theta)=h\left(\mathbf{A} \Theta_{1}+\mathbf{B} \Theta_{2}\right)
$$

In Eq.(21), $h$ refers to the maximum angular displacement of cam follower. Here, $h=22.5$. $\mathbf{A}$ and $\Theta$ refer to the coefficients matrix (dimensionless) and items matrix, respectively.

$$
\mathbf{A}=\left[\begin{array}{l}
a_{0} \\
a_{1} \\
a_{2} \\
a_{3} \\
a_{4} \\
a_{5} \\
a_{6} \\
a_{7}
\end{array}\right]^{\mathrm{T}}=\left[\begin{array}{r}
0.17522431 \\
-0.32001949 \\
0.24268741 \\
-0.15081006 \\
0.07478886 \\
-0.02817461 \\
0.00729689 \\
-0.00099837
\end{array}\right]^{\mathrm{T}} \quad \mathbf{B}=\left[\begin{array}{l}
b_{1} \\
b_{2} \\
b_{3} \\
b_{4} \\
b_{5} \\
b_{6} \\
b_{7}
\end{array}\right]^{\mathrm{T}}=\left[\begin{array}{l}
0 \\
0 \\
0 \\
0 \\
0 \\
0 \\
0
\end{array}\right]^{\mathrm{T}} \quad \boldsymbol{\Theta}_{1}=\left[\begin{array}{c}
1 \\
\cos \theta \\
\cos 2 \theta \\
\cos 3 \theta \\
\cos 4 \theta \\
\cos 5 \theta \\
\cos 6 \theta \\
\cos 7 \theta
\end{array}\right] \quad \boldsymbol{\Theta}_{2}=\left[\begin{array}{c}
\sin \theta \\
\sin 2 \theta \\
\sin 3 \theta \\
\sin 4 \theta \\
\sin 5 \theta \\
\sin 6 \theta \\
\sin 7 \theta
\end{array}\right]
$$

The errors of displacement, velocity and acceleration of this design case are:

$$
\left\{\begin{array}{l}
\varepsilon_{s}=8.06 \times 10^{-11} \\
\varepsilon_{v}=8.52 \times 10^{-8} \\
\varepsilon_{a}=7.54 \times 10^{-5}
\end{array}\right.
$$

According to the matrix $\mathbf{A}$, the weight of the coefficients of the sixth and seventh terms is little. The matrix $\mathbf{B}$ is null matrix, which indicates there is only cosine in Eq.(21). The main reason is that this displacement transfer function is centered exactly on $\theta=\pi$, which makes it possible to construct the displacement transfer function only by cosine. According to the magnitude of errors of displacement, velocity and acceleration, $n$ is equal to 7 for better accuracy and higher efficiency in this design case. 


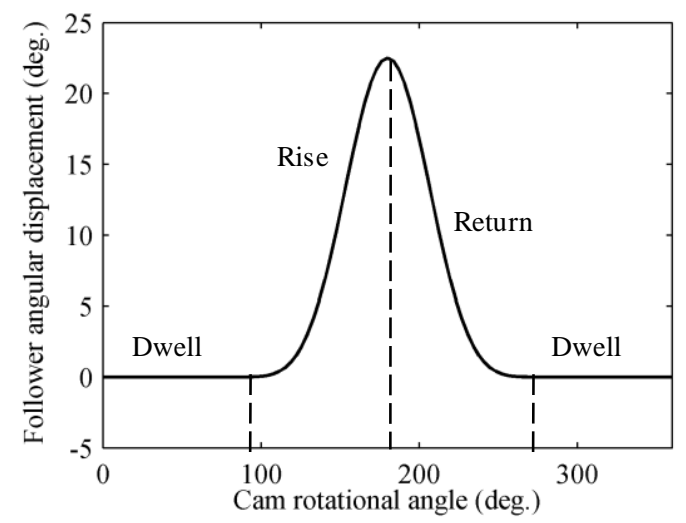

Fig.2. Motion law of the follower

Fig. 2 shows the angular displacement of the follower. In Fig.2, the curve is so smooth that the oscillating follower can work steadily. During the dwell period, the follower can remain still better, which shows the error between the actual displacement and the ideal displacement of the follower can be kept small using the displacement error function proposed in Section 3.

The cam profiles and the pressure angle can be achieved using the method in Section 4. Fig.3 and Fig.4 are the cam profiles and the curve of the pressure angle, respectively.

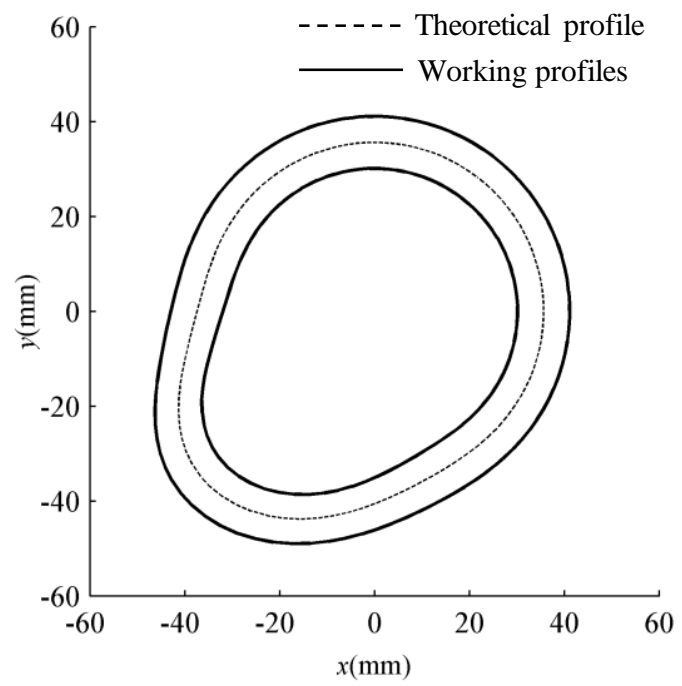

Fig. 3. Cam profiles

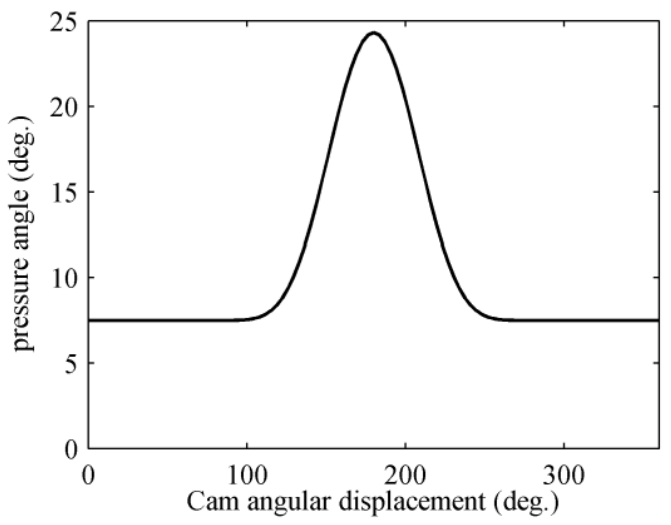

Fig. 4. Pressure angle

5.2 Continuous characteristic of displacement transfer function

When the cam is applied as to drive and control mechanism, the displacement transfer function is the key 
parameter. Generally, the output variable is displacement $s$ of the follower. The stability can be evaluated by the order of continuous derivative $\mathrm{d}^{p} s / \mathrm{d} t^{p}$. In Ref [24], the higher the order $p$ is, the smoother the motion curve of the follower is. It is proposed in Ref [9] that the higher order $p$ can reduce the vibration of the cam mechanism. Hence, the value $p$ is a key parameter in high-speed cam mechanism.

In order to compare with the performance of different cam curves, the kinematic characteristics of the follower should be normalized (dimensionless). Fig.5 shows 0 4 orders dimensionless kinematic characteristics (where, dimensionless $J=\mathrm{d}^{3} s / \mathrm{d} t^{3}, Q=\mathrm{d}^{4} s / \mathrm{d} t^{4}$ ).

(a)

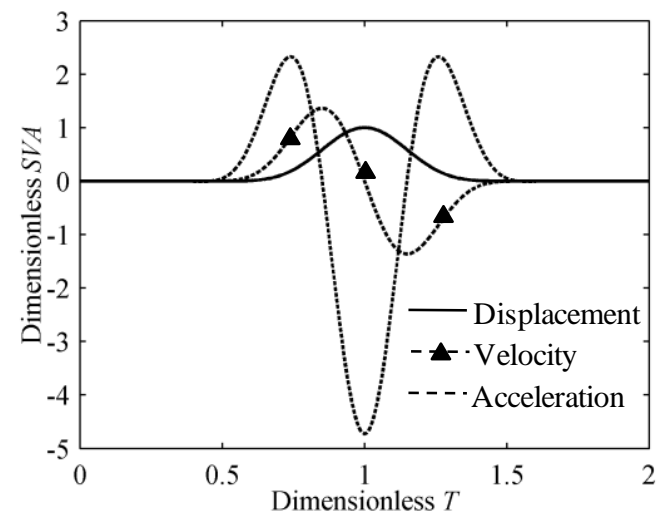

(b)

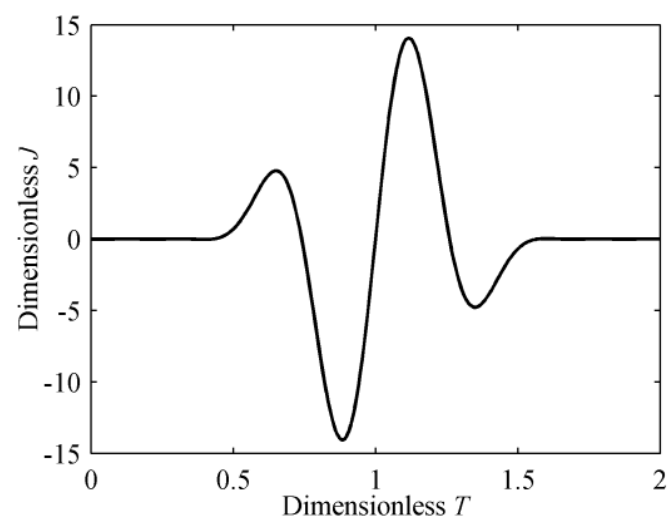

( c )

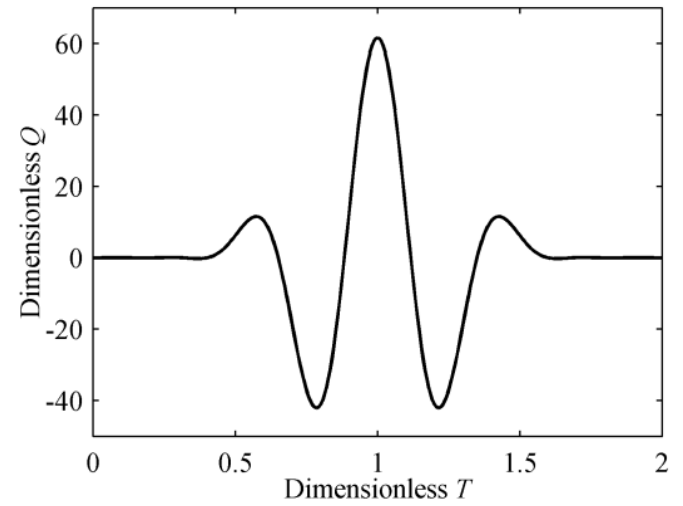

Fig.5. Dimensionless kinematic characteristics

According to the dimensionless displacement curve in Fig.5 (a), the displacement of the follower can meet the motion requirements in Table.2. When $p=4$, dimensionless $Q$ is continuous in Fig.4. The displacement transfer function is composed of some sine functions and cosine functions, so its higher derivative can still keep continuous. The results indicate that the proposed innovative method for designing a cam is feasible and the vibration of the cam mechanisms can be reduced by it.

\subsection{Dynamic torque}

In high-speed cam mechanisms, the dynamic torque is a major component of the torque of the cam mechanism. Without the friction between the cam and the roller, the balance torque of the cam mechanism can be written as follows and the detailed derivation is carried out in Appendix B:

$$
M=\left(m_{23} g r_{c} \sin \beta+F l_{2} \sin \beta+j_{2} \ddot{\beta}\right) \frac{\dot{\beta}}{\omega_{1}}
$$

where, $m_{23}$ is the mass of the roller (2) and the oscillating follower (3), $r_{c}$ refers to the distance between the center of mass and rotation center of the follower. $\omega_{1}$ is the rotational angular velocity of the cam, $F$ is the external load of the follower, $j_{2}$ refers to rotational inertia of the follower. $\beta$ refers to the angular displacement of the follower, 
the expression and its derivative can be written as: $\beta=\beta_{0}+S(\theta), \dot{\beta}=\frac{\mathrm{d} S(\theta)}{\mathrm{d} t}=\dot{S}$ and $\ddot{\beta}=\ddot{S}$.

The torque caused by the gravity and the external load is much less than the inertia moment in high speed cam mechanism [25]. Therefore, the dynamic torque of the cam mechanism can be simplified as:

$$
M_{d}=\frac{j_{2}}{\omega_{1}} \dot{S} \ddot{S}
$$

Substituting the dimensionless velocity and acceleration into Eq.(24), the simplified dynamic torque can be written as:

$$
M_{d}=A V\left(\frac{j_{2} h^{2}}{\omega_{1} t_{\mathrm{h}}^{3}}\right)
$$

where, $h$ refers to the maximum displacement of the follower, $t_{\mathrm{h}}$ refers to the time during rise period. In Eq.(25), all the parameters in brackets are unrelated to the motion law of the cam mechanism. $(A V)_{\mathrm{m}}$ is defined as the maximum value of $A V$, it can be used to evaluate the influence of the dynamic torque. Therefore, the motion law with a smaller $(A V)_{\mathrm{m}}$ should be designed to reduce the vibration of the cam mechanism. Fig.6 shows the dimensionless $A V$ of a Fourier series cam. The smooth curve of the dimensionless $A V$ is achieved and the maximum value $(A V)_{\mathrm{m}}=3.08$.

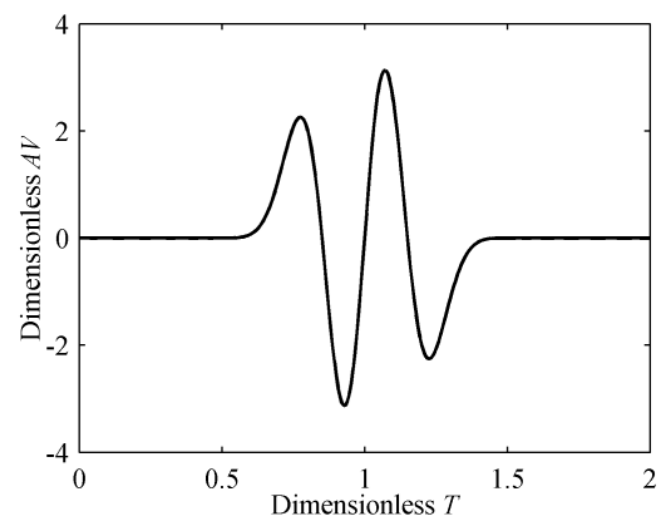

\subsection{Crossover impact}

Fig.6. $A V$ of Fourier series cam

There is a crossover impact in a cam pair with a geometric closure. As showed in Fig.7, when the acceleration of an oscillating follower is positive, the roller touches side $B$ of the driving cam. Because of the gap between the roller and the cam surface, the roller starts to cross the gap at point $M$ and impact side $A$ when the acceleration becomes zero from positive. This impact phenomenon is defined as the crossover impact and the point $M$ is called as the crossing point. 


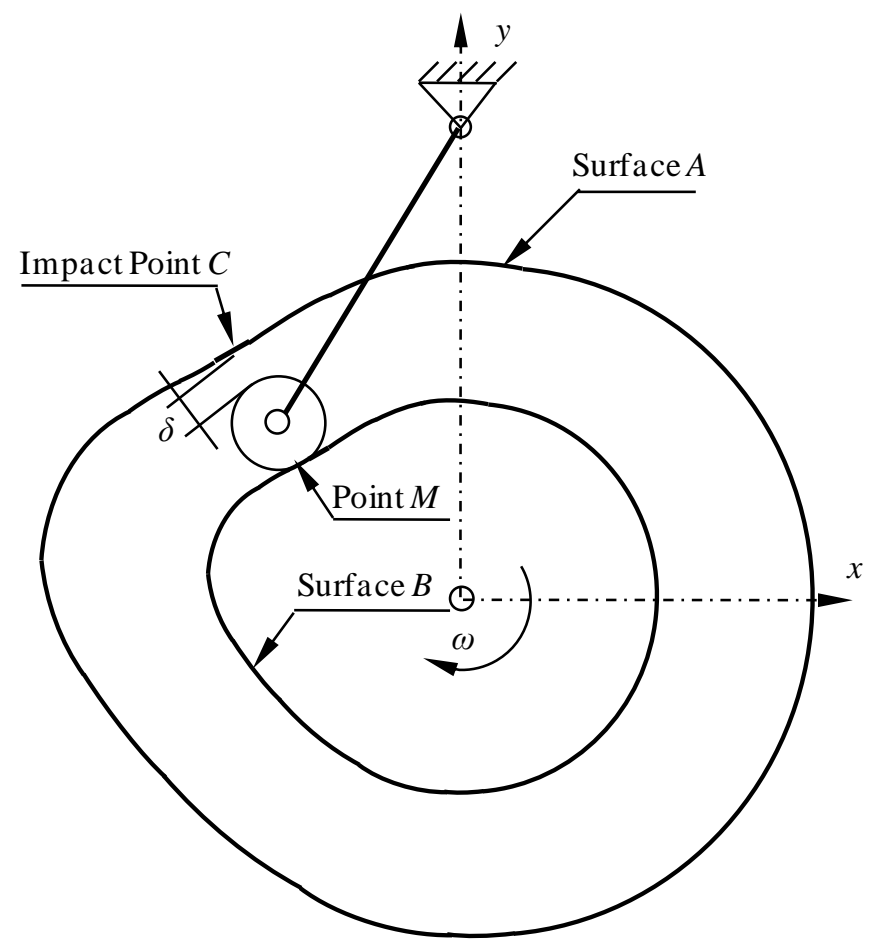

Fig.7. Cam mechanisms with geometric closure

$\delta$ refers to the gap between the roller and the cam working surface, and $\Delta t$ is the time required to cross the gap $\delta$. While $t=t_{0}$, the roller is separated from side $B$ at the velocity $v_{\mathrm{m}} . v_{\mathrm{c}}$ denotes the velocity of impact point on side $A$, and it still changes according to the velocity curve in Fig. 5(a). So the impact velocity, $v_{\text {imp }}$, can be written as:

$$
v_{\text {imp }}=v_{m}-v_{c}
$$

Based on Taylor series, $v_{\mathrm{c}}$ can be expanded as:

$$
v_{\mathrm{c}}=v\left(t_{0}+\Delta t\right)=v\left(t_{0}\right)+a\left(t_{0}\right) \Delta t+\frac{1}{2} j\left(t_{0}\right) \Delta t^{2}+\cdots
$$

where, $a\left(t_{0}\right)$ and $j\left(t_{0}\right)$ are the acceleration and the jerk respectively. According to the transmission mechanism of the cam mechanism, where $v\left(t_{0}\right)=v_{m}$ and $a\left(t_{0}\right)=0$. Substituting Eq.(27) into Eq.(26), $\Delta t$ is infinitesimal and its high order polynomial can be neglected. Therefore, the impact velocity can be written as:

$$
v_{\text {imp }}=-\frac{1}{2} j\left(t_{0}\right) \Delta t^{2}
$$

$\Delta t$ can be obtained by the following equation:

$$
\delta=\int_{t_{0}}^{t_{0}+\Delta t}\left(v_{m}-v\right) \mathrm{d} t
$$

Based on Taylor series, $\delta$ can be also expanded as:

$$
\delta=v_{m} \Delta t-\left[s\left(t_{0}+\Delta t\right)-s\left(t_{0}\right)\right]=v_{m} \Delta t-\left[s\left(t_{0}\right)+v\left(t_{0}\right) \Delta t+\frac{1}{2} a\left(t_{0}\right) \Delta t^{2}+\frac{1}{6} j\left(t_{0}\right) \Delta t^{3}+\cdots-s\left(t_{0}\right)\right]
$$

Substituting $v\left(t_{0}\right)=v_{m}$ and $a\left(t_{0}\right)=0$ into Eq.(30), $\delta$ can be written as:

$$
\delta=-\frac{1}{6} j\left(t_{0}\right) \Delta t^{3}
$$

Combining Eq.(28) and Eq.(31), the impact velocity can be written as:

$$
v_{\text {imp }}=\sqrt[3]{4.5\left|j\left(t_{0}\right)\right| \delta^{2}}
$$

Substituting the dimensionless velocity $\left(V_{\mathrm{imp}}=\frac{v_{\mathrm{imp}}}{h / t_{\mathrm{h}}}\right)$ and the dimensionless jerk ( $\left.J_{\text {imp }}=\frac{j_{\text {imp }}}{h / t_{\mathrm{h}}^{3}}\right)$ into Eq.(32), the dimensionless impact velocity can be written as: 


$$
V_{\mathrm{imp}}=\sqrt{4.5\left|J_{\mathrm{imp}}\right| \Delta^{2}}
$$

where, $\Delta$ refers to relative gap, namely, $\Delta=\delta / h$.

According to Eq.(32), the main factors that affect the impact velocity are the jerk at the crossing point and the gap between the roller and the cam working surface. The gap is a structural parameter which is unrelated to the motion law of the oscillating follower. Therefore, the jerk at the crossing point must be taken into consideration for designing the cam with geometric closure.

In this design case, when the follower arrives at the crossing point, the dimensionless time $T=0.8533$. At this point, the dimensionless jerk does not maximize, and its cube root $\sqrt[3]{J_{\mathrm{imp}}}=\sqrt[3]{12.98}=2.35$. For common motion law of follower, the jerk maximizes at the crossing point, which increases the crossover impact, such as a cycloid motion curve and a constant acceleration curve.

\subsection{Results discussion}

The velocity and the acceleration of the follower also have a great effect on the kinetic characteristics. A big momentum of the cam mechanism can lead to emergency stopping failure at high speed. The larger the acceleration of follower is, the bigger the contact force between the cam and the roller is, which will increase the wear between contact surfaces. Dimensionless characteristics of the Fourier series cam were analyzed by comparing with common cam curves in Table 3. Table 3 shows dimensionless kinematic characteristics of some cam mechanisms.

Table 3 Dimensionless kinematic characteristics

\begin{tabular}{cccccc}
\hline Cam curves & $V_{\mathrm{m}}$ & $A_{\mathrm{m}}$ & $p$ & $(A V)_{\mathrm{m}}$ & $\sqrt[3]{J_{\text {imp }}}$ \\
\hline Constant velocity curve & 1.00 & $\infty$ & 1 & - & - \\
Constant acceleration curve & 2.00 & 4.00 & 2 & 8.0 & $-\infty$ \\
Cosine acceleration curve & 2.00 & 6.28 & 3 & 8.16 & 3.41 \\
Modified cosine acceleration curve & 1.76 & 5.53 & 3 & 5.44 & 2.85 \\
Modified trapezoid acceleration curve & 2.00 & 4.89 & 3 & 8.05 & 3.95 \\
3-4-5 polynomial curve & 1.88 & 5.77 & 3 & 6.69 & 3.11 \\
4-5-6-7 polynomial curve & 2.19 & 7.51 & 4 & 10.75 & 3.75 \\
Modified constant velocity curve & 1.28 & 8.01 & 3 & 5.67 & 4.06 \\
Fourier series curve & 1.36 & 4.73 & $>4$ & 3.08 & 2.35 \\
\hline
\end{tabular}

The maximum velocity of a Fourier series curve is smaller than other cam curves excepted constant velocity curve in Table 3. The maximum acceleration is smaller than other cam curves excepted constant acceleration curve in Table 3. Therefore, the performance of emergency stopping will be better and the wear between contact surfaces will be decreased using a Fourier series cam curve.

The continuous characteristic of a Fourier series curve is better than other cam curves in Table 3. Its maximum dimensionless $A V=3.08$, which shows the fluctuation of the performance of a dynamic torque is little. The crossover impact velocity of a Fourier series cam mechanism with geometry closure is lower. Therefore, the vibration of a Fourier series cam at high speed is smaller than other cam curves in Table 3.

\section{Conclusions}

In this paper, an innovative design method for a high-speed cam has been presented. In the proposed method, the displacement transfer function of the cam mechanism has been developed using a Fourier series. The displacement error function has been constructed, which quantifies the variance between the actual displacement and the ideal displacement of the cam follower during the dwell period. The terms $n$ is equal to 7 calculated by Genetic Algorithm and the coefficients of the Fourier series have been obtained by the equality constraints and the inequality constraint equations. A smooth displacement curve designed has been obtained. The maximum velocity and acceleration of this cam follower are significantly smaller compared with other seven designs of cam curve 
shown in Table 3 excepted constant velocity curve or constant acceleration curve. Therefore, a superior performance for emergency stopping and less wear between contact surfaces in Fourier series cam mechanism at high speed can be achieved.

The comparison analysis of kinematic characteristics has indicated the order of continuous derivative of the Fourier series curve is higher, the characteristic value of its dynamic torque and its jerk at the impact point are smaller than other curves in Table 3. The results have confirmed that the proposed method can reduce the vibration of the cam mechanisms and its impact velocity is smaller than the others in Table 3.

\section{Acknowledgments}

The authors gratefully acknowledge the support of the National Science Foundation of China (NSFC) through Grants Nos.51275160 and Nos. 51305462.

\section{Appendix A}

When $n$ are equal to 6 , the coefficients matrix and errors are given as following:

$$
\mathbf{A}_{6}=\left[\begin{array}{l}
a_{0} \\
a_{1} \\
a_{2} \\
a_{3} \\
a_{4} \\
a_{5} \\
a_{6}
\end{array}\right]^{\mathrm{T}}=\left[\begin{array}{r}
0.18853888 \\
-0.33977648 \\
0.24702301 \\
-0.14186646 \\
0.06154644 \\
-0.01834257 \\
0.00290616
\end{array}\right]^{\mathrm{T}} \quad \mathbf{B}_{6}=\left[\begin{array}{l}
b_{1} \\
b_{2} \\
b_{3} \\
b_{4} \\
b_{5} \\
b_{6}
\end{array}\right]^{\mathrm{T}}=\left[\begin{array}{l}
0 \\
0 \\
0 \\
0 \\
0 \\
0
\end{array}\right]^{\mathrm{T}} \quad\left\{\begin{array}{l}
\varepsilon_{s}=2.7 \times 3 \quad \mathrm{P}^{\mathrm{T}} \\
\varepsilon_{v}=1.84 \times 10^{-6} \\
\varepsilon_{a}=8.98 \times 10^{-4}
\end{array}\right.
$$

When $n$ are equal to 8, the coefficients matrix and errors are given as following:

$$
\mathbf{A}_{8}=\left[\begin{array}{l}
a_{0} \\
a_{1} \\
a_{2} \\
a_{3} \\
a_{4} \\
a_{5} \\
a_{6} \\
a_{7} \\
a_{8}
\end{array}\right]^{\mathrm{T}}=\left[\begin{array}{r}
0.16431555 \\
-0.30326282 \\
0.23760410 \\
-0.15653911 \\
0.08528885 \\
-0.03731358 \\
0.01244394 \\
-0.00285573 \\
0.00032120
\end{array}\right]^{\mathrm{T}} \quad \mathbf{B}=\left[\begin{array}{l}
b_{1} \\
b_{2} \\
b_{3} \\
b_{4} \\
b_{5} \\
b_{6} \\
b_{7} \\
b_{8}
\end{array}\right]^{\mathrm{T}}=\left[\begin{array}{l}
0 \\
0 \\
0 \\
0 \\
0 \\
0 \\
0 \\
0
\end{array}\right]^{\mathrm{T}} \quad\left\{\begin{array}{l}
\varepsilon_{s}=7.34 \times 10^{-12} \\
\varepsilon_{v}=9.02 \times 10^{-9} \\
\varepsilon_{a}=1.19 \times 10^{-5}
\end{array}\right.
$$




\section{Appendix B}

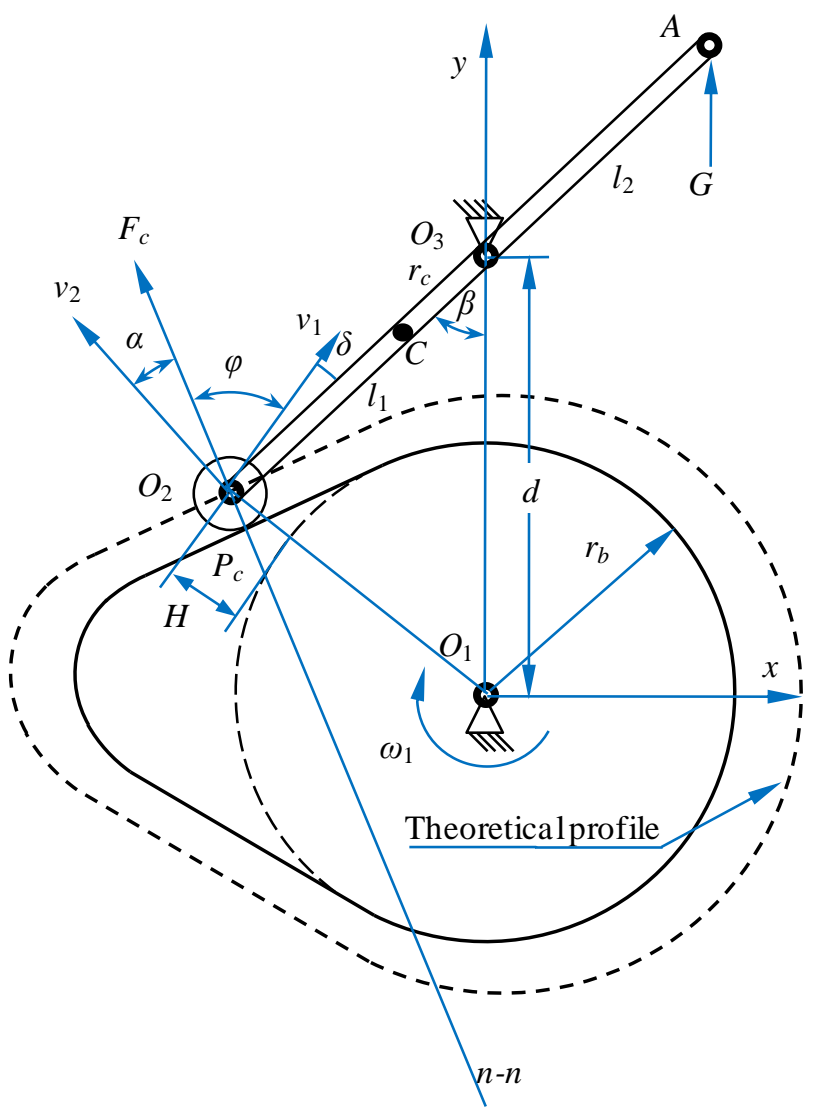

Fig.8. The force analysis of the cam mechanism

Fig. 8 show that the force analysis of the cam mechanism. Without the friction between the cam and the follower and between bearings, torque equilibrium equation of the follower can be written as:

$$
j_{2} \ddot{\beta}=F_{c} l_{1} \cos \alpha-m_{23} g r_{c} \sin \beta-F l_{2} \sin \beta
$$

Torque equilibrium equation of the cam can be written as:

$$
M=F_{c}\left(r_{b}+H\right) \cos \varphi
$$

where, $M$ refers to the balance torque of the rotating shaft of the cam.

According to meshing theory of the cam mechanism, the velocity along contact normal $n-n$ of the cam and the follower are equal:

$$
v_{1} \cos \varphi=v_{2} \cos \alpha
$$

where, $v_{1}=\omega_{1}\left(r_{b}+H\right), \quad v_{2}=\dot{\beta} l_{1}$

Substituting Eqs.(34)and (36) into Eq.(35), the balance torque of the rotating shaft of the cam can be written as:

$$
M=\left(m_{23} g r_{c} \sin \beta+F l_{2} \sin \beta+j_{2} \ddot{\beta}\right) \frac{\dot{\beta}}{\omega_{1}}
$$




\section{References}

[1] Kuang J H, Hsu C M, Hu C C. Dynamic behavior of globoidal cam systems with torque compensation mechanisms,J. Mechanism and Machine Theory. 45(2010):1201-1214.

[2] Lin K J, Huang C H, Liu Y L, et al. Influence of post-cam design of posterior stabilized knee prosthesis on tibiofemoral motion during high knee flexion,J. Clinical Biomechanics. 26(2011):847-852.

[3] CHANG Yong, YANG Fufu. Second Mechanism Synthesis Task of Disc Cam Mechanisms with Roller Follower Moving in Planar General Motion.,J. Chinese Journal of Mechanical Engineering. 46(2010):35-41.(In Chinese)

[4] J. Avsec, Z. Praunseis and M. Marcic. Nonlinear vibrational analysis of diesel valve gear,J. Journal of Vibroengineering. 13(2011):646-653

[5] Hrones J A. Analysis of dynamic force in a cam-driven system,J. Trans. ASME. 70(1948): 473-482

[6] Mitchell D B. Test on dynamic response of cam follower systems,J. Mechanical. Engineering. 72(1950): 467-471

[7] Hiroshi Makino. Mechanisms of automatic machinery,M. Beijing:Science Press, 1980.(In Chinese)

[8] PENG Guoxun, XIAO Zhengyang. Design of cam mechanisms of automatic machinery,M. Beijing: China Machine Press, 1990. (In Chinese)

[9] Jianping S, Zhaoping T. The Parametric Design and Motion Analysis about Line Translating Tip Follower Cam Mechanism Based on Model Datum Graph,J. Procedia Engineering. 23(2011):439-444.

[10] GE Zhenghao, FENG Tao, PENG Guoxun. General polynomial motion law with arbitrarily added local control conditions,J. Mechanical Science and Technology. 17(1998): 986-987. (In Chinese)

[11] Nguyen V T, Kim D J. Flexible cam profile synthesis method using smoothing spline curves,J. Mechanism and Machine Theory. 42(2007):825-838.

[12] K. Yoon, S.S. Rao, Cam motion synthesis using cubic splines,J. Transactions of the ASME. Journal of Mechanical Design. 115(1993): 441-446.

[13] SUN Shufeng, ZHOU Yiqi. Design of spatial cam based on non-uniform rational b-splines,J. Chinese Journal of Mechanical Engineering. 45(2009):125-129(In Chinese)

[14] Gao, Y.H,YANG Z.Q,ZHAO W.X, etal. Optimum design of cam curve of zoom system based on Zemax,J. Optik- International Journal for Light and Electron Optics. 124(2013): 6358-6362.

[15] Hsieh J F. Design and analysis of cams with three circular-arc profiles,J. Mechanism and Machine Theory. 45(2010):955-965

[16] Hsieh J F. Design and analysis of indexing cam mechanism with parallel axes,J. Mechanism and Machine Theory. 81(2014):155-165.

[17] Qiu H, Lin C J, Li Z Y, Ozaki H, Wang J, Yue Y. A universal optimal approach to cam curve design and its applications,J. Mechanism and Machine Theory. 40(2005):669-692.

[18] Fabien B.C. The design of dwell-rise-dwell cams with reduced sensitivity to parameter variation,J. Journal of the Franklin Institute. 332(1995):195-209.

[19] Wiederrich J L, Roth B. Dynamic Synthesis of Cams Using Finite Trigonometric Series,J. Journal of Engineering for Industry, Februay. 97(1975):287-293.

[20] Gutman A S. Synthesis of new cam profiles from frequency components,C. ASME paper 62-WA-64, 1962.

[21] J. Angeles, Synthesis of plane curves with prescribed local geometric properties using periodic splines, Computer-Aided-Design 15 (1983) 147-155.

[22] B.L. MacCarthy, N.D. Burns, An evaluation of spline functions for use in cam design, Proceedings of the Institution of Mechanical Engineering 199 (1985) 239-248.

[23] D.M. Tsay, C.O. Huey Jr., Cam motion synthesis using spline functions, ASME Journal of Mechanisms, Transmissions, and Automation Design 110 (1988) 161-165.

[24] Koster M P, Vibrations of cam mechanisms,M. London:Mecmillan Pubishers Ltd., 1974

[25] Zhang Ce, Machinery dynamics (second edition), M, Beijing: Higher Education Press, 2010 